\title{
Retinoic Acid Regulates Germ Gene Transcription In Vitro and Spermatogenesis in Testicular Organ Culture
}

\author{
Ni Hong ${ }^{1,2}$, Mingyou $\mathrm{Li}^{1}$, Beiping $\mathrm{He}^{3}$ and Yunhan Hong ${ }^{1,4 \star}$
}

${ }^{1}$ Department of Biological Sciences, National University of Singapore, Singapore

${ }^{2}$ DUKE-NUS Graduate Medical School, Singapore

${ }^{3}$ Department of Anatomy, Yong Loo Lin School of Medicine, National University of Singapore, Singapore

${ }^{4}$ Key Laboratory of Protein Chemistry and Developmental Biology of State Education Ministry of China, College of Life Sciences, Hunan Normal University, Changsha, 410081, China

\begin{abstract}
The adult testis is the male reproductive organ that produces sex hormones for sexual behaviours and sperm in a well-controlled process called spermatogenesis. Spermatogenesis involves self-renewal and differentiation of the male germ stem cells spermatogonia, culminating in the production of sperm for germline transmission. Retinoic acid (RA) is essential for fate decision and meiosis entry of mammalian germ cells. The microphthalmia-associated transcription factor (Mitf) is a newly identified transcriptional activator of germ genes in the fish medaka (Oryzias latipes). Here we report an essential role of RA on Mitf-activated germ gene expression and spermatogenesis. Medaka germ gene promoters DAZ, DND and VAS were found to contain RA-responsive elements and to exhibit little Mitf-activated transcriptional activity in response to RA treatment in both medaka ES cells and male germ stem cells. A procedure for testicular organ culture was established, which allows for test-tube spermatogenesis over a 21-day period. By the ordered testicular architecture, cellular morphology and VAS-driven GFP expression, different spermatogenic stages were identifiable on testicular cross-sections. In organ culture, RA treatment generated testes completely free of spermatogonia, and reduced VAS-driven GFP expression in spermatogonia but enhanced its expression in meiotic and post-meiotic germ cells. Therefore, RA represses Mitf-activated germ gene expression in stem cell cultures and differentially regulates germ gene expression depending upon the stages of spermatogenesis, and represses spermatogonial stem cell maintenance in cultured testes of medaka as a lower vertebrate model. These results demonstrate that RA plays a highly conserved role in germ cell development from fish to mammals.
\end{abstract}

Keywords: Gene expression; Fertility; Retinoids; Organ culture; Spermatogenesis

Abbreviations: mitf: Microphthalmia-Associated Transcription Factor; dpt: Day(S) Post Transfection

\section{Introduction}

In diverse animal species, adult gonads, the testis in male and ovary in female, are the reproductive organ that produces sexual hormone for secondary sex characteristics and gametes, sperm in male and eggs in female, for germline transmission. Germline development commences with specification of primordial germ cells (PGCs) early in embryonic development and culminates in the production of gametes in the adult gonads. PGCs migrate into the developing gonad and become gonocytes, which subsequently become germ stem cells, oogonia and spermatogonia depending on the gonadal sex. Oogonia and spermatogonia undergo differentiation through meiosis to produce eggs in the ovary and sperm in the testis [1]. Germ cells maintain totipotency throughout development [2]. Although gametes are highly specialized cells, they demonstrate this potential upon fertilization to generate a new organism [3]. Understanding of germ cell-specific molecular regulators is a key for dissecting the molecular mechanisms underlying germ cell development and totipotency.

Studies in model organisms have identified both evolutionarily conserved and divergent regulators of germ cell development [4], and revealed the importance of transcriptional repression of somatic fates and post-transcriptional control of gene expression in regulating germ cell development [2,5]. Remarkably, many evolutionarily conserved germ genes - including dazl, dead end and vasa - encode RNA-binding proteins. However, how these germ genes are regulated for expression at the transcriptional level has remained essentially unexplored. One exception is the recent identification of microphthalmiaassociated transcription factor (Mitf) as a transcription activator of germ gene expression in medaka [6]. Mitf is a basic helix-loop-helix/ leucine zipper protein and acts as a master regulator of melanocyte development $[7,8]$. Mitf plays a critical role also in the development of retinal pigment epithelium [9-12] and has been documented in human pigment diseases $[13,14]$.

Retinoic acid (RA) has been demonstrated to play important roles in diverse processes including patterning of the tetrapod limb [15] and the central nervous system [16], formation of blood vessel and bone [17], osteogenesis [18] and specification of cardiomyocytes [19] and liver cells [20]. RA is the active derivative of vitamin A (or retinol), which is synthesized intracellularly and transported to the nucleus where it initiates transcription of target genes [21]. RA functions via binding to retinoic acid receptors (RARs), which bind preferentially as heterodimers with the retinoid $\mathrm{X}$ receptor (RXR) to the RA response element (RARE) in the regulatory regions of target genes. The RARE is a direct repeat (DR) of GGTCA spaced by 1 7 nucleotides with TGACC. These receptors are ligand-dependent transcription factors. An RAR:RXR heterodimer binds to the RA-responsive element (RARE) to regulate transcription.

Recent studies have revealed that RA plays essential roles in various

*Corresponding author: Yunhan Hong, Department of Biological Sciences National University of Singapore, 14 Science Drive 4, Singapore 117543, Tel: +6565162915; Fax: +65-67792486; E-mail: dbshyh@nus.edu.sg

Received November 02, 2012; Accepted December 14, 2012; Published December 17, 2012

Citation: Hong N, Li M, He B, Hong Y (2012) Retinoic Acid Regulates Germ Gene Transcription In Vitro and Spermatogenesis in Testicular Organ Culture. Biol Syst Open Access 1:103. doi:10.4172/2329-6577.1000103

Copyright: $\odot 2012$ Hong N, et al. This is an open-access article distributed under the terms of the Creative Commons Attribution License, which permits unrestricted use, distribution, and reproduction in any medium, provided the original author and source are credited. 
processes and aspects of germline development. RA signaling determines the germ cell fate in mice [22]. RA induces the transdifferentiation of myoblasts into premeiotic Stra8-positive cells [23]. More profoundly, RA has been best implicated in controlling meiotic entry. In mice, germ cells within the developing gonad follow a sexually dimorphic pathway. Germ cells in the ovary enter into the meiotic prophase during embryogenesis, whereas germ cells in the testis arrest in $G_{0}$ of mitotic cell cycle and do not commence meiosis until after birth. Retinoic acid (RA), synthesized from the dietary vitamin retinol or vitamin A, regulates meiotic entry in both male and female germ cells. In female mice, meiosis is initiated soon after gonadal sex determination by 12.5 days post coitum $(\mathrm{dpc})$. This initiation can be blocked by RA antagonists or by disruption of the RA-regulated gene Stra8 [24,25]. In contrast to the fetal ovary, gonocytes in a 12.5 -dpc fetal testis enter the $G_{0}$ phase of the cell cycle and do not divide until after birth. The exit of male gonocytes from the cell cycle is not a consequence of their inability to respond to RA, because the gonocytes of a fetal testis without RA enter meiosis when incubated with RA [22]. It is also not the result of a lack of RA synthesis, as ALDH1A2, the rate-limiting enzyme in RA biosynthesis, is expressed in the mesonephros of both sexes [22]. The testicular RA concentration at $12.5 \mathrm{dpc}$ is only $20 \%$ of the ovarian concentration, because the testis expresses CYP26B1, an enzyme that converts RA to inactive metabolites [25]. In fetal Cyp26b1-null mice, the testicular RA level is elevated 3-fold and gonocytes initiate meiosis at $12.5 \mathrm{dpc}$ [26]. Therefore, the RA concentration, but not the genetic sex of the gonocytes, determines the initiation of meiosis in mice.

RA regulates meiotic entry also in chicken [27] and human [28]. The importance of RA signaling in mitotic-to-meiotic transition is well-exemplified by the identification of stra8, one of the genes stimulated by RA, which is a marker of premeiotic mouse germ cells $[29,30]$. In vivo, RA promotes meiosis via the down-regulation of Nanos2, whereas FGF9 inhibits meiosis through the upregulation of Nanos2 [31]. In vitro, RA promotes proliferation of PGCs from mouse [32] and chicken [33], and induces differentiation of embryonic stem (ES) cells from mouse and fish [34,35].

RA is required also for the maintenance of adult mammalian spermatogenesis. Upon vitamin A withdrawal in adult rodents, germ cells fail to enter into meiosis and remain undifferentiated, and spermatogenesis is disrupted by the loss of differentiated germ cells, which accompanies the accumulation of spermatogonia within the seminiferous epithelium [36]. These results support a notion that RA sets a balance between germ stem cell self-renewal and differentiation via controlling meiotic entry in both oogenesis and spermatogenesis of mammals.

This study was aimed at determining the role of RA in regulating germ gene expression and germ cell development in lower vertebrates. We use the fish medaka (Oryzias latipes) as a model to study stem cells [37-40] germ cells [40-42] and sexual development [43,44]. We have recently identified Mitf as a first transcriptional activator of germ genes [6]. Here we show that RA suppresses Mitf-activated germ gene expression in stem cell cultures and exhausts the spermatogonial stem cell pool in organ culture.

\section{Materials and Methods}

\section{Fish}

Work with fish followed the guidelines on the Care and Use of Animals for Scientific Purposes of the National Advisory Committee for Laboratory Animal Research in Singapore and approved by this committee (permit number 27/09). Medaka (Oryzias latipes) strain $i^{1}$ and transgenic line $\mathrm{Vg}$ were maintained under an artificial photoperiod of 14 -h/10-h light/darkness at $26^{\circ} \mathrm{C}$ as described $[40,45,46]$. In medaka, sex maturation occurs until 3 months post hatching. Breeding males and females measuring $\sim 4 \mathrm{~cm}$ in length at $6 \sim 7$ months were used for organ culture and analysis.

\section{Gene identification and RT-PCR}

The medaka RARs and RXRs are annotated in the genome sequence (http://www.ensembl.org/index.html). Total RNA was isolated by using the Trizol reagent (Invitrogen) and used for cDNA synthesis by using oligo $(\mathrm{dT})_{25}$ and the MMLV reverse transcriptase (Invitrogen) as described [47]. RT-PCR was run in $25-\mu$ l volume containing $10 \mathrm{ng}$ of cDNA for 30 cycles (for $\beta$-actin) and 35 cycles (RARs and RXRs) of $10 \mathrm{~s}$ at $94^{\circ} \mathrm{C}, 15 \mathrm{~s}$ at $58^{\circ} \mathrm{C}$ and $60 \mathrm{~s}$ at $72^{\circ} \mathrm{C}$. Information on genes and PCR primers used were provided in table 1 . The PCR products were separated on $1.2 \%$ agarose gels and documented with a bioimaging system (Synoptics). The PCR products were sequenced by using the BigDye Terminator Cycle Sequencing Ready Reaction Kit and analyzed on the ABI 377 sequencer (Perkin-Elmer, USA).

\section{Reporter and effector plasmids}

Six luciferase (Luc) reporter plasmids and the effector plasmid pXmitf used in this study have been described $[6,48]$. pXmitf expresses the Xiphophorus mitfm [49]. Plasmid DNA was prepared by using the Qiagen Midi-preps kit (Qiagen).

\section{Cell Culture and luciferase reporter assay}

The medaka ES cell line MES1 and spermatogonial line SG3 were maintained on gelatin-coated tissue culture plastic ware in medium ESM4 [34] and transfected as described [50]. For reporter assays, cells were transfected in 96-well plates as described [6]. In some experiments, cells were treated with all-trans RA (Sigma). For this, RA stock $(5 \mathrm{mM})$ dissolved in dimethyl sulfoxide (DMSO) was added to pre-warmed ESM4 to a final concentration of $2 \mu \mathrm{M}$. Controls were treated only with DMSO as vehicle. The RA-containing ESM4 was applied to cells after incubation with the transfection reagent-DNA complex. At 3 or

\begin{tabular}{|c|c|c|c|}
\hline Gene & Accession Number & Names and Sequences of Primers & Size (bp) \\
\hline rara1 & ENSORLG00000004373 & $\begin{array}{l}\text { a1F: AAGTCCTCAGGGTACCACTATGGT } \\
\text { a1R:GGCGCTCAGCAGTCCCGTCTCTGC }\end{array}$ & 720 \\
\hline rara2 & ENSORLG00000008502 & $\begin{array}{l}\text { a2F: ATGTTTGACTGCATGGACGTGCTG } \\
\text { a2R:GGATGGGAAAGTTTCTTGATGGGC }\end{array}$ & 564 \\
\hline rary1 & ENSORLG00000007861 & $\begin{array}{l}\text { Y1F: GTACCCAGCTCTCCGTCTCCACCT } \\
\text { Y1R: CAGCTGAACACGGTGGTCTGAGCT }\end{array}$ & 450 \\
\hline rary2 & ENSORLG00000015382 & $\begin{array}{l}\text { Y2F: CCTCACATGTTTCCCCGCATGCTG } \\
\text { Y2R:CGCCGCCTTCTTCTGCACGGCCGC }\end{array}$ & 360 \\
\hline $\operatorname{rxr} \beta$ & ENSORLG00000007020 & 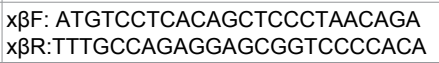 & 300 \\
\hline rxry & ENSORLG00000016690 & $\begin{array}{l}\text { xyF: AGTGTCAGCAGCTCCGAGGACATC } \\
\text { xyR: ACAGTACTGACAGCGGTTCCGCTG }\end{array}$ & 270 \\
\hline mitf1 & ENSORLG00000013461 & $\begin{array}{l}\text { m1F: GTAGATTACATCCGCAAGCTG } \\
\text { m1R: ACAGCCCTGCTCGTTTTCATCCAT }\end{array}$ & 501 \\
\hline dazl & NM_001104799 & $\begin{array}{l}\text { dzF: AATACCTTCATCATCCTCACCTC } \\
\text { dzR: AAGTGAAGGAACGTTTGACC }\end{array}$ & 328 \\
\hline dnd & NM_001164516 & $\begin{array}{l}\text { ddF: CAGAGCAAGGTGGTGAACTTG } \\
\text { ddR: CACCTTGTAGGTGAAGTACAG }\end{array}$ & 966 \\
\hline vasa & NM_001104676 & $\begin{array}{l}\text { vsF: CGTCCAGCACGTGGTGAACT } \\
\text { vsR: TTCCGGACTTGAACGTGGG }\end{array}$ & 486 \\
\hline p-detint & NM_001104808 & $\begin{array}{l}\text { MA1: TTTCAACAGCCCTGCCATGTAC } \\
\text { MA2: CCTCCAATCCAGACAGAGTATT }\end{array}$ & 650 \\
\hline
\end{tabular}

1) PCR was run with a common annealing temperature of $58^{\circ} \mathrm{C}$ for all primers used

Table 1: Primers used for RT-PCR analysis ${ }^{1)}$. 
4 days post transfection, cells were measured for luciferase activity by using the Dual-Glo ${ }^{\mathrm{TM}}$ Luciferase assay kit (Promega) and measured on Glomax $^{\mathrm{TM}} 96$ Microplate Luminometer (Promega). The reading from pGL3-basic was considered as background, and that from pTAluc treated as unit activity. Relative luciferase activity from vectors was normalized to that of pTAluc.

\section{Testicular organ culture}

Testes were dissected aseptically from 9 Vg adult medaka males. A medaka has two testes, one in the left abdomen and the other in the right abdomen. Intact gonads were seeded in 24 -well plates, one testis per well containing $0.5 \mathrm{ml}$ of ESM2 medium with or without $10 \mu \mathrm{M}$ of RA. Medium ESM2 has proven suitable for deriving and maintaining medaka ES cells $[34,35,51,52]$ and testicular cell lines including SG3 capable of test-tube sperm production [47]. ESM2 is similar in osmolarity to media capable of supporting male germ cell proliferation and differentiation in primary culture of medaka testis [53] and primary spermatocytes [54].

\section{Testicular cryosections}

Testes were fixed in $4 \%$ paraformaldehyde in phosphate-buffered saline (PBS) at $4^{\circ} \mathrm{C}$ for $1 \mathrm{~h}$ and subjected to cryosectioning as described [46]. The sections were stained for nuclei with DAPI $(1 \mu \mathrm{g} / \mathrm{ml})$ for 10 min at room temperature, washed three times in PBS and mounted for microscopy [55]. Germ cells were identified by transgenic GFP expression from the germ cell-specific VAS promoter. Different stages of germ cell development in the adult testis were recognized by relative intensities of GFP signal, cellular location and morphology within the ordered testicular structure. A total of 9 pairs of testes were subjected to detailed analyses by sectioning. Three testes were examined at 3,21 and 28 days of culture in the absence of RA. In parallel, three testes were analyzed at the same intervals of culture in the presence of RA.

\section{Microscopy and photography}

Tissue sections and cell cultures were observed under a Zeiss Axiovert2 invert microscope and/or Axiovert200 upright microscope by using a Zeiss AxioCam MRc digital camera [35,55]

\section{Statistics}

Statistical analyses were calculated by using Graphad Prism v4.0. Data consolidated were presented as mean \pm s.d., and p-values were calculated by using nonparametric Student's $t$-test as described [46].

\section{Results}

\section{Consensus RARE site present in medaka germ gene promoters}

Since RA regulates gene expression through binding to RAREs, we wanted to examine whether the consensus RARE sequence was present in the promoter region of medaka germ genes. We chose dazl, dnd and vasa that have been characterized as germ cell markers [55-59]. In particular, VAS, a $5.1-\mathrm{kb}$ vasa promoter is able to drive germ cell specific GFP expression [58]. There are 1 2 full RARE sites and 7 12 RARE half sites within the $4.2-\mathrm{kb}$ dazl promoter (DAZ), 5.4-kb dnd promoter (DND) and 5.1-kb VAS, respectively (Figure 1A), revealing them as putative RA target genes.

\section{Expression of RA signaling genes in medaka stem cells and testes}

In mammals, there are three RA receptors (RAR $\alpha, R A R \beta$ and $\operatorname{RAR} \gamma)$ and three retinoid $\mathrm{X}$ receptors $(\mathrm{RXR} \alpha, \operatorname{RXR} \beta$ and $\operatorname{RXR} \gamma)$ that initiate the signaling through binding by RA. These receptors may exist as duplicate in teleosts due to an ancient whole genome duplication event in the fish lineage. In medaka, four RARs and two RXRs are annotated in the genome (http://www.ensembl.org/index.html). We examined the RNA expression of five of these receptors by RT-PCR analysis. All the 5 genes exhibited detectable expression in the medaka ES cell line MES1, spermatogonial cell line SG3, testis and ovary (Figure 1B). This indicates that the RA signaling pathway is active in medaka ES cells and male germ stem cells in culture as well as the adult gonad.

\section{RA suppresses Mitf-activated germ gene transcription in stem cell cultures}

Previously we have shown that Mitf is expressed in medaka germ cells in vivo and capable of activating germ gene transcription in vitro [6], coincident with the presence of multiple putative E-box consensus element, to which Mitf binds to regulate target gene transcription, in the three germ gene promoters (Figure 1A). For reporter assays, these promoters were individually inserted upstream of the fire fly luciferase (luc) in the promoterless construct pGL3-basic, resulting in pDAZluc, pDNDluc and pVASluc respectively (Figure 1C). We made use of SG3 and MES1 cells for transfection and reporter assay. SG3 is a male germ stem cell line from the adult medaka spermatogonia and expresses germ genes dazl and vasa [55]. MES1 is a medaka ES cell line capable of chimera formation $[45,46,60]$. Both cell lines are suitable to study germ gene expression by reporter assay [6].

In both cell lines, the three germ gene promoters alone exhibited activities approximately 60 120 times the minimal promoter TA

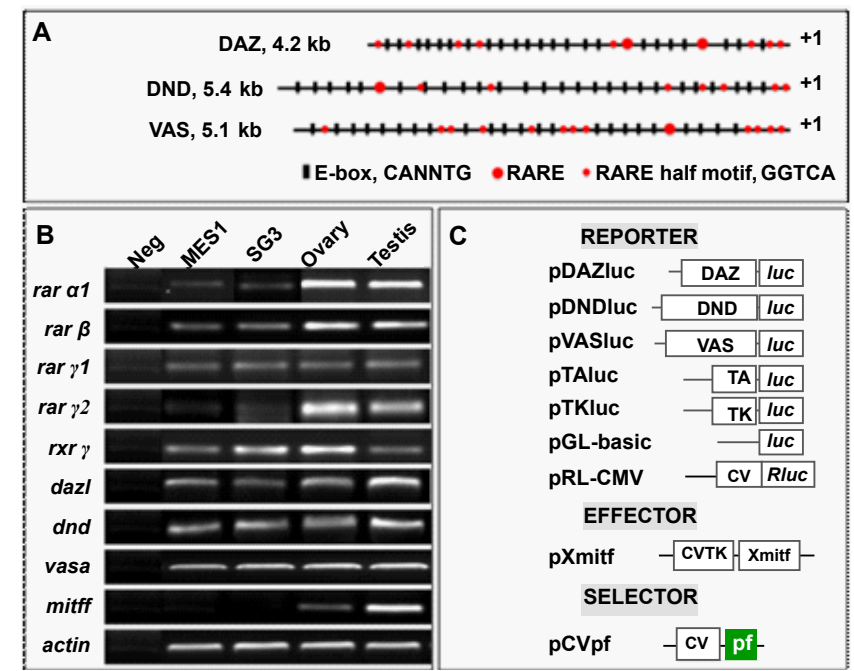

(A) Promoters of the medaka dazl, dnd and vasa. The first letter of the initiation codon ATG is defined as +1 , and the upstream sequence is shown as minus. Putative consensus $E$ box sequences are shown in box, and RA-responsive element (RARE) and its half site are shown in circle.

(B) RT-PCR analysis of gene expression. Actin was used as a loading control. MES1 RNA instead of its cDNA was used as the template, which served as negative controls (Neg).

(C) Reporter and effector vectors. DAZ, DND, VAS, promoters of the medaka dazl, dnd and vasa;

$\mathrm{CV}$ : the human cytomegalovirus immediate early enhancer/promoter; TA: minimal promoter containing a TATA-box; TK: human herpes simplex virus thymidine kinase promoter; CVTK: CV enhancer in combination with TK promoter; luc: firefly luciferase; Rluc: renilla luciferase as the internal control; pGL3-basic: promoterless vector as the negative control; Xmitf: cDNA for the M is form of the Xiphophorus mitf

Figure 1: Reporter system and gene expression profile. 
(Figures 2A and 2B). We used pXmitf as effector, a construct expressing the Xiphophorus mitfm, because Xmitf has been well-documented [61] and proven to be efficient to induce melanocyte in MES1 [49] and SG3 cells [62]. Upon cotransfection with pXmitf, the activity increased by 3.6, 3.8 and 4.4 fold in SG3 cells (Figure 2A), by 5.9, 6 and 5.5 in MES1 cells (Figure 2B) fold for DAZ, DND and VAS, respectively. When RA was added to cell cultures co-transfected with pXmitf and a reporter plasmid, the transcription activity was reduced by $3 \sim 5$ fold to the basal level in both SG3 and MES1 (Figures 2A and 2B). Clearly, RA abolishes Mitf-activated germ gene transcription in medaka ES cells and adult male germ cells in culture, suggesting that the three germ genes are targets of the RA signaling.

\section{RA abolishes the spermatogonial cell pool}

To analyze the effect of RA treatment on adult germ cells, we dissected intact $\mathrm{Vg}$ testes and seeded them for organ culture in medium ESM2. The Vg testis shows strong GFP expression in the periphery rich in spermatogonia, spermatogenic cysts but little GFP signal in the efferent duct containing sperm (Figures $3 \mathrm{~A}$ and $3 \mathrm{~B}$ ). Within the testis under these conditions, spermatogenesis occurs during the first three weeks of organ culture in the absence and presence of RA, because different stages of spermatogenic germ cells, ranging from spermatogonia to motile sperm, were released from the intact testes via the efferent duct (Figures $3 \mathrm{C}-3 \mathrm{~F}$ ). These results suggest that the adult testis under organ culture conditions used retains the capability of spermatogenesis in vitro.

Spermatogenesis diminished gradually and essentially ceased after 3 weeks of organ culture in the presence of RA. Since RA stimulates meiotic entry in chicken and mammals $[25,27,28]$, we examined the testicular structure on cryosections. In medaka, the adult testis consists of cysts of germ cells, each cyst is surrounded by Sertoli cells and comprises of synchronously developing germ cells. Cysts of different spermatogenic stages are arranged in such a way so that the spermatogonial cysts are at the testicular periphery, followed by the cysts of primary spermatocytes, secondary spermatocytes and spermatids, with sperm being matured at the testicular center and stored in the lumen located at the center. Besides a peripheral location, spermatogonia exist as singlets, doublets or small clusters from the periphery towards the center, exhibit relatively weaker nuclear staining and the strongest VAS-driven GFP expression. When spermatogenesis proceeds, the number of male germ cells per cyst increases, nuclear staining becomes more intense and VAS-driven GFP expression decreases (Figures $4 \mathrm{~A}$ and $4 \mathrm{~B}$ ). These features in combination with the sequential arrangement of different-stages of spermatogenic cysts allow for unambiguous definition of individual spermatogenesis stages, and thus a precise analysis of normal and abnormal spermatogenesis in cultured testes. After 3 days of culture, the control testis maintained in the absence of RA exhibited normal distribution of all stages of spermatogenesis ranging from spermatogonia to sperm (Figures $4 \mathrm{~A}$ and $4 \mathrm{~A}$. The testis maintained for 3 days in the presence of RA displayed a similar structure but a remarkable reduction in the number of spermatogonia (compare figures $4 \mathrm{~B}$ and $4 \mathrm{~B}^{\prime}$ with figures $4 \mathrm{~A}$ and $4 \mathrm{~A}$. Thus, in short-term testicular culture, RA reduces the number of spermatogonia.

After 21 days of culture in the absence of RA, the testis still exhibited many spermatogonia and early meiotic germ cells (e.g. primary spermatocytes), which distributed mostly in the peripheral region, as well as late stages of spermatogenic cells (spermatids and sperm), which were found outside the peripheral region (Figures 5A-
5D). In contrast, after 21 days of culture in the presence of RA, the testis became completely exhausted of spermatogonia, leading to a peripheral region essentially free of GFP-positive germ cells (Figures 5E and $5 \mathrm{~F}$ ). After 28 days of RA treatment, the number of germ cells was remarkably reduced, leading to areas of merely somatic cells not only in the peripheral but also central regions of the testis (Supplementary Figure S1). Taken together, RA treatment in extended organ culture

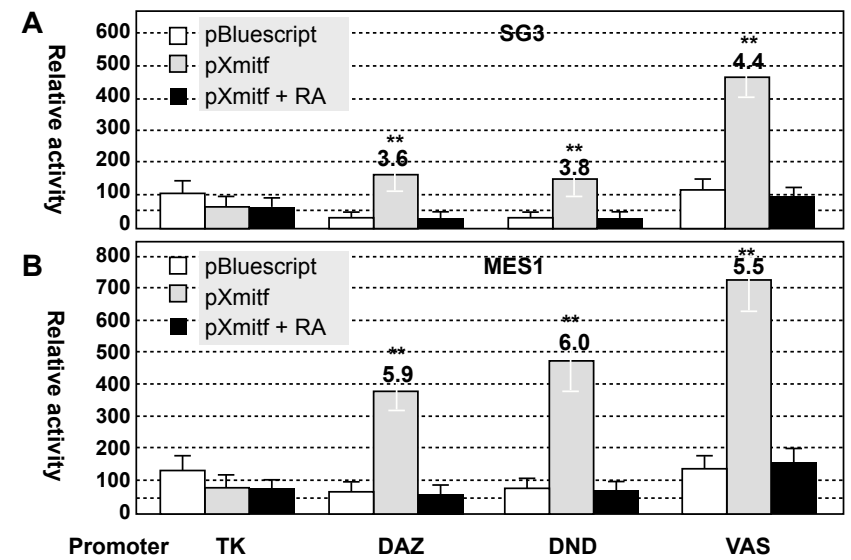

(A) Reporter assays by pXmitf transfection in the adult male germ cell line SG3. (B) Reporter assays by pXmitf transfection in ES cell line MES1. The reading from pTAluc transfection was regarded as unit activity for normalization. Relative activities are means \pm s.d. from three independent experiments of transfection in triplicate each, which were normalized to vectors sizes (Figure 1B). Values above columns are fold changes comparison to cotransfection with pCVpf as carrier DNA. ${ }^{* *}$ very significant difference at $p \leq 0.01$

Figure 2: RA Suppresses Mitf-Activate Germ Gene Transcription.

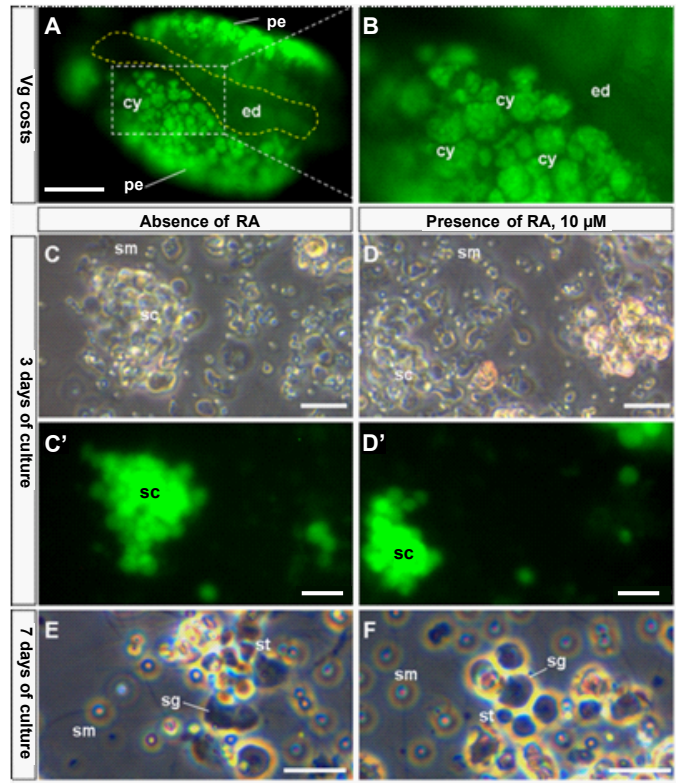

(A) Intact $\mathrm{Vg}$ testis showing strong GFP expression in the periphery (pe) and spermatogenic cysts (cy) but little signal in the efferent duct (ed) containing mature sperm. (B) Large magnification of the area framed in (A), highlighting spermatogenic cysts (cy). (C-F) Spermatogenesis until one week of testicular organ culture with and without RA, showing individual spermatogonia (sg), spermatocytes (sc), spermatids (st) and sperm (sm) released from intact testes in culture. Scale bars, $200 \mu \mathrm{m}$.

Figure 3: Spermatogenesis in Testicular Organ Culture. 

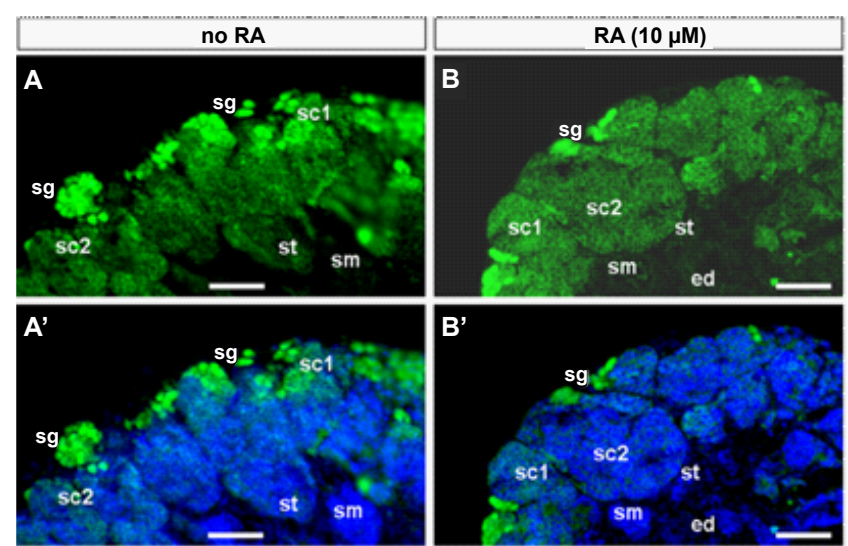

$\mathrm{Vg}$ testicular cryosections were stained with DAPI for nuclei (blue) and microscopically analyzed under different optics.

A and A Cryosection of one intact testis at day 3 of organ culture in the absence of RA, showing relative abundance of different stages of spermatogenesis.

B' and B' Cryosection of the other intact testis from the same fish at day 3 of organ culture in the presence of $10 \mu \mathrm{M} \mathrm{RA}$, showing a significant decrease in the number of spermatogonia.

ed: efferent; sg: spermatogonia; sc1: primary spermatocytes; sc2: secondary spermatocytes; st: spermatids; sm: sperm. Scale bars, $100 \mu \mathrm{m}$.

Figure 4: RA affects spermatogenesis in short-term organ culture.

disrupts the maintenance of spermatogonial stem cell pool.

\section{RA exerts different effects on germ gene expression depending on stages}

The fact that RA inhibits Mitf-mediated germ gene expression in cell culture provoked us to examine its effect on testicular germ gene expression in organ culture. Compared to the control testis maintained in the absence of RA (Figure 4A), the intensity of GFP signal altered markedly after 3 days of RA treatment: It became weaker in spermatogonia but stronger in meiotic cells (primary and secondary spermatocytes; Figure 4B). Elevated expression in late meiotic cells (spermatids) was most evident in the testes following RA treatment for 21 days (Compare Figures 5C and 5E). Furthermore, RA treatment prevented the loss of GFP expression in sperm (Compare Figures 5C and $5 \mathrm{E}$ ). Taken together, VAS-driven transgenic gene expression is suppressed in spermatogonial stem cells but elevated in meiotic and post-meiotic male germ cells by RA treatment in testicular organ culture, suggesting that RA exerts different effects on germ gene expression depending on stages of germ cell development.

\section{Discussion}

In mammals, the regulatory role of RA has recently been well documented in germline development, ranging from fate decision over proliferation to meiosis entry $[22,25,27,28,32,33]$. In this study, we provide first evidence that RA regulates germ gene expression and germ cell development also in medaka as a lower vertebrate model, suggesting that the RA signaling plays a highly conserved role in controlling germline development from fish to mammals.

In vitro in the absence of somatic cells, RA promotes proliferation of PGC cultures from mouse [32] and chicken [33]; in vivo, RA stimulates differentiation and inhibits self-renewal of gonadal germ stem cells of mouse [31], human [28] and chicken [27]. The mechanism underlying the effect of RA on proliferation in vitro or differentiation in vivo of germ stem cells remains unknown. A difference in response to RA treatment might be due to a difference between PGCs and spermatogonia in nature and assay condition. In this study, the testicular organ culture system reveals that RA stimulates differentiation and inhibits selfrenewal of spermatogonial stem cells. This suggests that male germ cells within dissected testes in organ culture resemble those of intact testes in live animals in response to RA treatment, underscoring the usefulness of organ culture system for studying germ cell proliferation and differentiation.

In this study, we show that all the three germ genes possess the $\mathrm{RA}$ responsive element in their regulatory regions and that, more importantly, RA abolishes activation of the germ genes' transcription by Mitf. These results suggest that RA signaling may be directly involved in regulating germ gene transcription. Since our reporter assay has used stem cell culture and RA is a strong inducer of stem cell differentiation in medaka $[34,35]$, it is also likely that RA may prevent Mitf-activated germ gene expression through induced differentiation of stem cells. RA works through binding to its receptors. We reveal that RA receptors are indeed expressed in both cell lines used for reporter assays. Future work will determine whether RA receptors are differentially expressed at different stages of medaka germ cell development of both sexes and required for the regulation of germ gene expression.

These results show that transcription of germ genes in cell culture is controlled by two opposing mechanisms, one is activation by Mitf and the other is silencing by RA. Furthermore, the negative transcriptional effect of RA is dominant over the activating effect of Mitf. A similar observation has also been made with the mouse oct4, an octamerbinding transcription factor that plays a key role in the maintenance of pluripotency and exhibits pluripotency-specific expression in ES cells
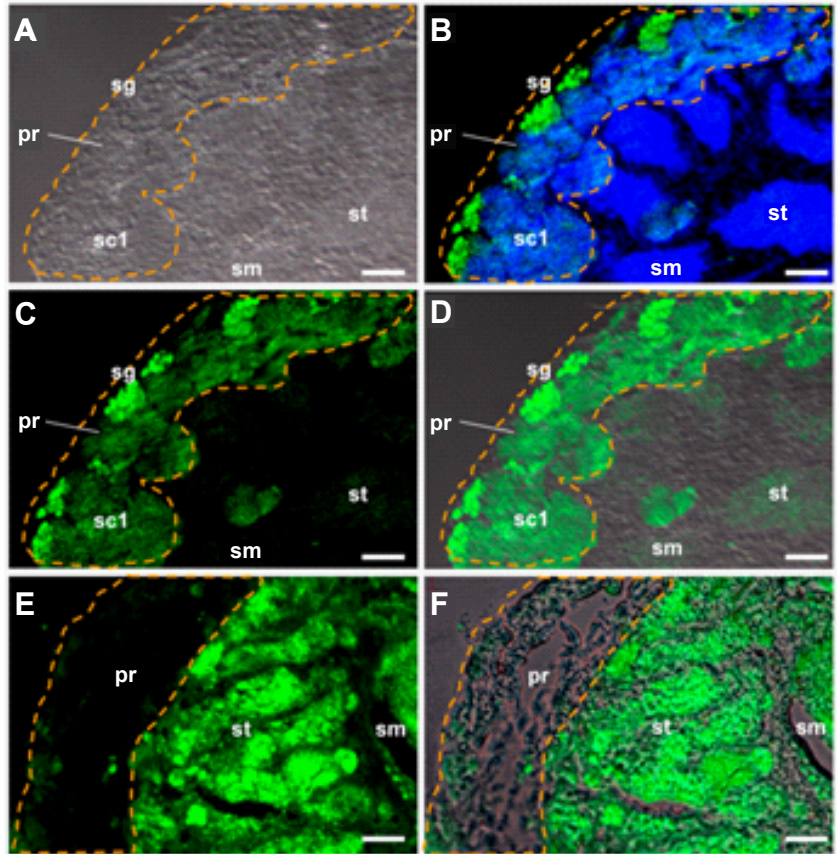

(A-D) Cryosection of one intact testis at day 21 of organ culture in the absence of RA, showing many spermatogenic cells including spermatogonia and primary spermatocytes in the peripheral region ( $p r$, outline).

( $E$ and $F$ ) Cryosection of the other intact testis from the same fish at day 3 of organ culture in the presence of $10 \mu \mathrm{M}$ RA, showing the absence of spermatogenic cells in the peripheral region ( $\mathrm{pr}$, outline), and a relatively stronger GFP signal in spermatids and sperm.

sg: spermatogonia; sc1: primary spermatocytes; sc2: secondary spermatocytes; st: spermatids; sm: sperm. Scale bars, $50 \mu \mathrm{m}$.

Figure 5: RA affects spermatogenesis in extended organ culture. 
and germline [63]. Oct4 is down-regulated by RA via several RARE half-sites in the proximal promoter [64].

We observe that RA does reduce VAS-driven GFP reporter expression in spermatogonia in testicular organ culture, which is in accordance with RA-mediated suppression of Mitf-activated germ gene expression in stem cell culture. It has been shown that endogenous expression occurs at the highest level in spermatogonia, declines remarkably during meiosis and becomes hardly detectable in postmeiotic stages for germ genes examined in this study, namely dazl and vasa [55], dnd [56] and mit [6].

In medaka, it has been reported that expression of the vasa RNA is at the highest level in spermatogonia and reduced in meiosis and post-meiotic phase on testicular sections [55], and that vasa promoterdriven GFP expression occurs at the highest level also in mitotic and early meiotic germ cells in the peripheral cysts of the whole testis [65]. Results in this study collaborate and extend these reports by revealing that on testicular sections, VAS-driven GFP expression is the highest in spermatogonia, remains abundant in primary spermatocyte and reduces in meiotic and post-meiotic germ cells.

An interesting observation obtained in this study is that RA reduces GFP reporter expression from the vasa promoter in spermatogonial cells but enhances GFP expression in meiotic and post-meiotic male germ cells. This finding is consistent with the reports that vasa is essential for proliferation, differentiation and meiotic progress of germ cells in mouse [66] and human [67]. In mice, it has been reported that RA as well as stem cell factor increases meiotic entry, upregulates expression of meiotic genes stra8 (master regulator of meiosis), $d m c 1$ (early meiotic marker essential for meiotic recombination), bmp6, btg4, $d z i p 11$ and $p h f 7$, and downregulates expression of stemness genes ( $p c g f 2$, pcgf6 and numb) and spermatogonial markers (egr 2, pole, ptn, tex 16 and $z f p m 2$ ), which are known to turn off at the onset of meiosis [68]. Most recently, the medaka 5.1-kb VAS promoter used for driving GFP expression has been reported to contain up to 11 positive and negative regulatory regions, which exert opposing mechanisms underlying vasa transcription [48]. This study identifies one RARE and 12 copies of the RARE half site. Future work is needed to determine the precise region(s) on which RA acts to regulate stage-specific vasa expression.

\section{Acknowledgments}

We thank Jiaorong Deng for fish breeding. $\mathrm{Dr}$. J. Altschmied (Düsseldorf, Germany) for kindly providing plasmid pXmitf, and Yovita Ida Purwanti for par of work. This research was supported by the National Research Foundation of Singapore (NRF-CRP7-2010-03 and NRF-CRP 002-082).

\section{References}

1. Wylie C (1999) Germ cells. Cell 96: 165-174.

2. Pesce M, Gross MK, Schöler HR (1998) In line with our ancestors: Oct-4 and the mammalian germ. Bioessays 20: 722-732.

3. Seydoux G, Braun RE (2006) Pathway to totipotency: lessons from germ cells. Cell 127: 891-904.

4. Ewen-Campen B, Schwager EE, Extavour CG (2010) The molecular machinery of germ line specification. Mol Reprod Dev 77: 3-18.

5. Cinalli RM, Rangan P, Lehmann R (2008) Germ cells are forever. Cell 132 $559-562$

6. Zhao H, Li M, Purwanti YI, Liu R, Chen T, et al. (2012) Mitf is a transcriptional activator of medaka germ genes in culture. Biochimie 94: 759-767.

7. Hodgkinson CA, Moore KJ, Nakayama A, Steingrímsson E, Copeland NG, et al. (1993) Mutations at the mouse microphthalmia locus are associated with defects in a gene encoding a novel basic-helix-loop-helix-zipper protein. Cell 74: 395-404.
8. Vance KW, Goding CR (2004) The transcription network regulating melanocyte development and melanoma. Pigment Cell Res 17: 318-325.

9. Hallsson JH, Favor J, Hodgkinson C, Glaser T, Lamoreux ML, et al. (2000) Genomic, transcriptional and mutational analysis of the mouse microphthalmia locus. Genetics 155: 291-300.

10. Lister JA, Robertson CP, Lepage T, Johnson SL, Raible DW (1999) nacre encodes a zebrafish microphthalmia-related protein that regulates neural-crestderived pigment cell fate. Development 126: 3757-3767.

11. Steingrímsson E, Moore KJ, Lamoreux ML, Ferré-D’Amaré AR, Burley SK et al. (1994) Molecular basis of mouse microphthalmia (mi) mutations helps explain their developmental and phenotypic consequences. Nat Genet 8: 256263.

12. Tsujimura T, Morii E, Nozaki M, Hashimoto K, Moriyama Y, et al. (1996) Involvement of transcription factor encoded by the mi locus in the expression of c-kit receptor tyrosine kinase in cultured mast cells of mice. Blood 88: 12251233.

13. Smith SD, Kelley PM, Kenyon JB, Hoover D (2000) Tietz syndrome (hypopigmentation/deafness) caused by mutation of MITF. J Med Genet 37: 446-448.

14. Tassabehji M, Newton VE, Read AP (1994) Waardenburg syndrome type 2 caused by mutations in the human microphthalmia (MITF) gene. Nat Genet 8: 251-255.

15. Yashiro K, Zhao X, Uehara M, Yamashita K, Nishijima M, et al. (2004) Regulation of retinoic acid distribution is required for proximodistal patterning and outgrowth of the developing mouse limb. Dev Cell 6: 411-422.

16. Hernandez RE, Putzke AP, Myers JP, Margaretha L, Moens CB (2007) Cyp26 enzymes generate the retinoic acid response pattern necessary for hindbrain development. Development 134: 177-187.

17. Hayashida Y, Kawamura T, Hori-e R, Yamashita I (2004) Retionic acid and its receptors are required for expression of aryl hydrocarbon receptor mRNA and embryonic development of blood vessel and bone in the medaka fish, Oryzias latipes. Zoolog Sci 21: 541-551.

18. Spoorendonk KM, Peterson-Maduro J, Renn J, Trowe T, Kranenbarg S, et al. (2008) Retinoic acid and Cyp26b1 are critical regulators of osteogenesis in the axial skeleton. Development 135: 3765-3774.

19. Keegan BR, Feldman JL, Begemann G, Ingham PW, Yelon D (2005) Retinoic acid signaling restricts the cardiac progenitor pool. Science 307: 247-249.

20. Negishi T, Nagai Y, Asaoka Y, Ohno M, Namae M, et al. (2010) Retinoic acid signaling positively regulates liver specification by inducing wnt2bb gene expression in medaka. Hepatology 51: 1037-1045.

21. Ross SA, McCaffery PJ, Drager UC, De Luca LM (2000) Retinoids in embryona development. Physiol Rev 80: 1021-1054.

22. Bowles J, Knight D, Smith C, Wilhelm D, Richman J, et al. (2006) Retinoid signaling determines germ cell fate in mice. Science 312: 596-600.

23. Jia W, Cheng D, Chen S, Lei L, Wang H (2011) Retinoic acid induces myoblasts transdifferentiation into premeiotic Stra8-positive cells. Cell Biol Int 35: 365-372.

24. Anderson EL, Baltus AE, Roepers-Gajadien HL, Hassold TJ, de Rooij DG, et al. (2008) Stra8 and its inducer, retinoic acid, regulate meiotic initiation in both spermatogenesis and oogenesis in mice. Proc Natl Acad Sci U S A 105: 1497614980.

25. Koubova J, Menke DB, Zhou Q, Capel B, Griswold MD, et al. (2006) Retinoic acid regulates sex-specific timing of meiotic initiation in mice. Proc Natl Acad Sci U S A 103: 2474-2479.

26. MacLean G, Li H, Metzger D, Chambon P, Petkovich M (2007) Apoptotic extinction of germ cells in testes of Cyp26b1 knockout mice. Endocrinology 148: $4560-4567$

27. Smith CA, Roeszler KN, Bowles J, Koopman P, Sinclair AH (2008) Onset of meiosis in the chicken embryo; evidence of a role for retinoic acid. BMC Dev Biol 8: 85

28. Childs AJ, Cowan G, Kinnell HL, Anderson RA, Saunders PT (2011) Retinoic Acid signalling and the control of meiotic entry in the human fetal gonad. PLoS One 6: e20249.

29. Bouillet P, Oulad-Abdelghani M, Vicaire S, Garnier JM, Schuhbaur B, et al. 
Citation: Hong N, Li M, He B, Hong Y (2012) Retinoic Acid Regulates Germ Gene Transcription In Vitro and Spermatogenesis in Testicular Organ Culture. Biol Syst Open Access 1:103. doi:10.4172/2329-6577.1000103

(1995) Efficient cloning of cDNAs of retinoic acid-responsive genes in P19 embryonal carcinoma cells and characterization of a novel mouse gene, Stra1 (mouse LERK-2/Eplg2). Dev Biol 170: 420-433.

30. Hogarth CA, Mitchell D, Evanoff R, Small C, Griswold M (2011) Identification and expression of potential regulators of the mammalian mitotic-to-meiotic transition. Biol Reprod 84: 34-42.

31. Barrios F, Filipponi D, Pellegrini M, Paronetto MP, Di Siena S, et al. (2010) Opposing effects of retinoic acid and FGF9 on Nanos2 expression and meiotic entry of mouse germ cells. J Cell Sci 123: 871-880.

32. Farini D, Scaldaferri ML, Iona S, La Sala G, De Felici M (2005) Growth factors sustain primordial germ cell survival, proliferation and entering into meiosis in the absence of somatic cells. Dev Biol 285: 49-56.

33. Yu M, Guan K, Zhang C (2011) The promoting effect of retinoic acid on proliferation of chicken primordial germ cells by increased expression of cadherin and catenins. Amino Acids 40: 933-941.

34. Hong Y, Winkler C, Schartl M (1996) Pluripotency and differentiation of embryonic stem cell lines from the medakafish (Oryzias latipes). Mech Dev 60: $33-44$

35. Yi M, Hong N, Hong Y (2009) Generation of medaka fish haploid embryonic stem cells. Science 326: 430-433.

36. Li H, Palczewski K, Baehr W, Clagett-Dame M (2011) Vitamin A deficiency results in meiotic failure and accumulation of undifferentiated spermatogonia in prepubertal mouse testis. Biol Reprod 84: 336-341.

37. Hong N, Li Z, Hong Y (2011) Fish stem cell cultures. Int J Biol Sci 7: 392-402.

38. Hong $Y(2011)$ Fishing fish stem cells and nuclear transplants. Int J Biol Sci 7: 390-391.

39. Li M, Shen Q, Xu H, Wong FM, Cui J, et al. (2011) Differential conservation and divergence of fertility genes boule and dazl in the rainbow trout. PLoS One 6: e15910.

40. Li M, Hong N, Xu H, Yi M, Li C, et al. (2009) Medaka vasa is required for migration but not survival of primordial germ cells. Mech Dev 126: 366-381.

41. Nakamura S, Kobayashi K, Nishimura T, Higashijima S, Tanaka M (2010) Identification of germline stem cells in the ovary of the teleost medaka. Science 328: $1561-1563$

42. Xu H, Li M, Gui J, Hong Y (2010) Fish germ cells. Sci China Life Sci 53: 435446 .

43. Matsuda M, Nagahama Y, Shinomiya A, Sato T, Matsuda C, et al. (2002) DMY is a Y-specific DM-domain gene required for male development in the medaka fish. Nature 417: 559-563.

44. Nanda I, Kondo M, Hornung U, Asakawa S, Winkler C, et al. (2002) A duplicated copy of DMRT1 in the sex-determining region of the $Y$ chromosome of the medaka, Oryzias latipes. Proc Natl Acad Sci U S A 99: 11778-11783.

45. Hong N, Chen S, Ge R, Song J, Yi M, et al. (2012) Interordinal chimera formation between medaka and zebrafish for analyzing stem cell differentiation. Stem Cells Dev 21: 2333-2341.

46. Hong N, Li M, Zeng Z, Yi M, Deng J, et al. (2010) Accessibility of host cell lineages to medaka stem cells depends on genetic background and irradiation of recipient embryos. Cell Mol Life Sci 67: 1189-1202.

47. Hong Y, Liu T, Zhao H, Xu H, Wang W, et al. (2004) Establishment of a normal medakafish spermatogonial cell line capable of sperm production in vitro. Proc Natl Acad Sci U S A 101: 8011-8016.

48. Li M, Guan G, Hong N, Hong Y (2012) Multiple regulatory regions control the transcription of medaka germ gene vasa. Biochimie.

49. Béjar J, Hong Y, Schartl M (2003) Mitf expression is sufficient to direct differentiation of medaka blastula derived stem cells to melanocytes. Development 130: 6545-6553.

50. Hong Y, Chen S, Gui J, Schartl M (2004) Retention of the developmental pluripotency in medaka embryonic stem cells after gene transfer and long-term drug selection for gene targeting in fish. Transgenic Res 13: 41-50.

51. Hong Y., Schartl, Manfred (1996) Establishment and growth responses of early medakafish (Oryzias latipes) embryonic cells in feeder layer-free cultures. Mol Mar Biol Biotechnol 5: 93-104.

52. Li Z, Bhat N, Manali D, Wang D, Hong N, et al. (2011) Medaka cleavage embryos are capable of generating ES-like cell cultures. Int J Biol Sci 7: 418425

53. Song M, Gutzeit HO (2003) Primary culture of medaka (Oryzias latipes) testis: a test system for the analysis of cell proliferation and differentiation. Cell Tissue Res 313: 107-115.

54. Sasaki T, Watanabe A, Takayama-Watanabe E, Suzuki M, Abe H, et al. (2005) Ordered progress of spermiogenesis to the fertilizable sperm of the medaka fish, Oryzias latipes, in cell culture. Dev Growth Differ 47: 87-97.

55. Xu H, Li Z, Li M, Wang L, Hong Y (2009) Boule is present in fish and bisexually expressed in adult and embryonic germ cells of medaka. PLoS One 4: e6097.

56. Liu L, Hong N, Xu H, Li M, Yan Y, et al. (2009) Medaka dead end encodes a cytoplasmic protein and identifies embryonic and adult germ cells. Gene Expr Patterns 9: 541-548.

57. Shinomiya A, Tanaka M, Kobayashi T, Nagahama Y, Hamaguchi S (2000) The vasa-like gene, olvas, identifies the migration path of primordial germ cells during embryonic body formation stage in the medaka, Oryzias latipes. Dev Growth Differ 42: 317-326.

58. Tanaka M, Kinoshita M, Kobayashi D, Nagahama Y (2001) Establishment of medaka (Oryzias latipes) transgenic lines with the expression of green fluorescent protein fluorescence exclusively in germ cells: a useful model to monitor germ cells in a live vertebrate. Proc Natl Acad Sci U S A 98: 2544-2549.

59. Xu H, Li M, Gui J, Hong Y (2007) Cloning and expression of medaka daz during embryogenesis and gametogenesis. Gene Expr Patterns 7: 332-338.

60. Hong Y, Winkler C, Schartl M (1998) Production of medakafish chimeras from a stable embryonic stem cell line. Proc Natl Acad Sci U S A 95: 3679-3684.

61. Altschmied J, Delfgaauw J, Wilde B, Duschl J, Bouneau L, et al. (2002) Subfunctionalization of duplicate mitf genes associated with differential degeneration of alternative exons in fish. Genetics 161: 259-267.

62. Thoma EC, Wagner TU, Weber IP, Herpin A, Fischer A, et al. (2011) Ectopic expression of single transcription factors directs differentiation of a medaka spermatogonial cell line. Stem Cells Dev 20: 1425-1438.

63. Boiani M, Schöler HR (2005) Regulatory networks in embryo-derived pluripotent stem cells. Nat Rev Mol Cell Biol 6: 872-884.

64. Schoorlemmer J, van Puijenbroek A, van Den Eijnden M, Jonk L, Pals C, et al. (1994) Characterization of a negative retinoic acid response element in the murine Oct4 promoter. Mol Cell Biol 14: 1122-1136.

65. Zhao H, Hong N, Lu W, Zeng H, Song J, et al. (2012) Fusion gene vectors allowing for simultaneous drug selection, cell labeling, and reporter assay in vitro and in vivo. Anal Chem 84: 987-993.

66. Tanaka SS, Toyooka Y, Akasu R, Katoh-Fukui Y, Nakahara Y, et al. (2000) The mouse homolog of Drosophila Vasa is required for the development of male germ cells. Genes Dev 14: 841-853.

67. Medrano JV, Ramathal C, Nguyen HN, Simon C, Reijo Pera RA (2012) Divergent RNA-binding proteins, DAZL and VASA, induce meiotic progression in human germ cells derived in vitro. Stem Cells 30: 441-451.

68. Pellegrini M, Filipponi D, Gori M, Barrios F, Lolicato F, et al. (2008) ATRA and $\mathrm{KL}$ promote differentiation toward the meiotic program of male germ cells. Cell Cycle 7: 3878-3888 